\title{
Tolerance of Dispersion-Managed Soliton Transmission to the Shape of the Input Pulses
}

\author{
V. S. Grigoryan, G. M. Carter, and C. R. Menyuk
}

\begin{abstract}
We showed that long-distance transmission is affected by the source pulse tails. Fast tail dropoff dramatically improves the transmission. We found that the tails must be at least $20 \mathrm{~dB}$ down $50 \mathrm{ps}$ from the peak in order to successfully transmit signals over $20000 \mathrm{~km}$ at $20 \mathrm{Gbit} / \mathrm{s}$ with anomalous path average dispersion. While tails that are $15 \mathrm{~dB}$ down $50 \mathrm{ps}$ from the peak are sufficient to transmit data at $10 \mathrm{Gbit} / \mathrm{s}$ over $20000 \mathrm{~km}$ with anomalous path average dispersion, the tails must be down at least $25 \mathrm{~dB} 50 \mathrm{ps}$ from the peak to transmit data with slightly normal path average dispersion.
\end{abstract}

Index Terms-Optical fiber communication, optical solitons, optical fiber dispersion, optical propagation in nonlinear media.

\section{INTRODUCTION}

$\mathbf{O}$ $\mathrm{NE}$ of the major impairments in long-distance, high-datarate, dispersion-managed soliton (DMS) systems is the interpulse interaction between adjacent pulses. Previous study of this problem was mostly focused on the soliton-soliton interactions [1]-[5]. A notable exception is [6], where the interaction of return-to-zero pulses was considered. However, in real systems the initial puls differs from the final soliton shape. The pulses pass through a complex transient regime before transforming into solitons [7], and propagation in the transient regime can be as long as $10000 \mathrm{~km}$. Our analysis shows that when the initial pulses have slowly dropping tails, the interpulse interaction in the transient regime can be significantly stronger than the interpulse interaction in the soliton regime. The transient interpulse interaction can be so strong that it completely destroys the whole pulse train at distances much shorter than the transient regime. In this case, a true DMS propagation never appears.

We note that source noise by itself can limit the transmission distance [8], but it is not a major factor in our experiments [7].

In the present letter, we study both theoretically and experimentally the tolerance of long-distance DMS transmission in a recirculating fiber loop to the magnitude of the input pulse tails. We find the minimum extinction ratio that is required for signals to propagate beyond the transient regime at $10 \mathrm{Gbit} / \mathrm{s}$ in the anomalous dispersion regime, at $10 \mathrm{Gbit} / \mathrm{s}$ in the slightly normal dispersion regime, and at $20 \mathrm{Gbit} / \mathrm{s}$ in the anomalous dispersion regime.

Manuscript received March 6, 2000; revised May 11, 2000.

The authors are with the Department of Computer Science and Electrical Engineering, University of Maryland Baltimore County, Baltimore, MD 21228-5398 USA (e-mail: grigorya@umbc.edu; carter@umbc.edu; menyuk@umbc.edu).

Publisher Item Identifier S 1041-1135(00)07450-4.

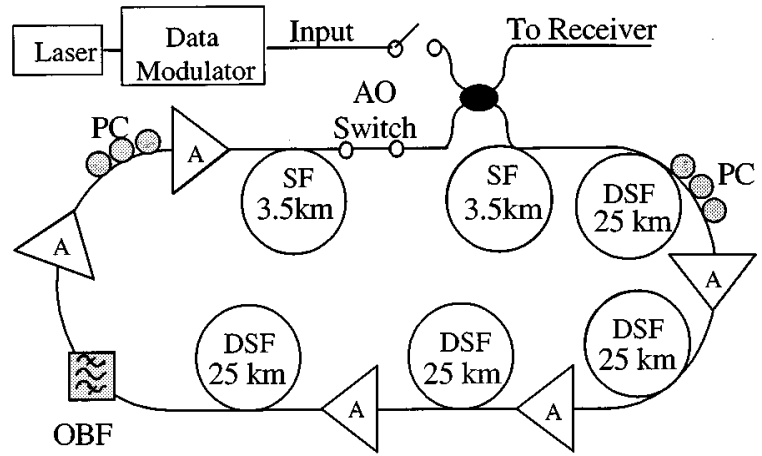

Fig. 1. Recirculating fiber loop setup.

\section{SYSTEM CHARACTERISTICS}

Our experimental setup is shown in Fig. 1. The dispersion map consists of $100 \mathrm{~km}$ of dispersion-shifted fiber with a normal dispersion of $-1.2 \mathrm{ps} / \mathrm{nm} \cdot \mathrm{km}$ followed by $7 \mathrm{~km}$ of the standard fiber with an anomalous dispersion of $16.7 \mathrm{ps} / \mathrm{nm} \cdot \mathrm{km}$. By detuning the central wavelength of the signal pulses we were able to vary the path-averaged dispersion from 0.03 $\mathrm{ps} / \mathrm{nm} \cdot \mathrm{km}$ (anomalous) to about $-0.01 \mathrm{ps} / \mathrm{nm} \cdot \mathrm{km}$ (normal). The details of the experimental setup have been reported in [9]. We model pulse propagation in the loop using the modified nonlinear Schrödinger equation

$$
\begin{aligned}
i \frac{\partial q}{\partial z}+\frac{1}{2}[D(z)-i b(z)] \frac{\partial^{2} q}{\partial t^{2}}+\frac{i}{6} d \frac{\partial^{3} q}{\partial t^{3}} & +|q|^{2} q \\
& =i g(z) q+\hat{F}(z, t) .
\end{aligned}
$$

Here, the pulse envelope $q$ is normalized as $q=$ $E\left(n_{2} \omega_{0} L_{D} / A_{\mathrm{eff}} c\right)^{1 / 2}$ where $E$ is the electric field envelope, $n_{2}=2.6 \times 10^{-16} \mathrm{~cm}^{2} / \mathrm{W}$ is the Kerr coefficient, $\omega_{0}$ is the central frequency, $A_{\text {eff }}=47 \mu \mathrm{m}^{2}$ is the effective area, $c$ is the speed of light, $L_{D}=T_{0}^{2} / \beta_{0}^{\prime \prime}$ is the dispersion length, $T_{0}$ and $\beta_{0}^{\prime \prime}$ are characteristic scale time and scale dispersion, respectively. The distance $z$ is normalized as $z=Z / L_{D}$, where $Z$ is the physical distance, and the time $t$ is the retarded time normalized to $T_{0}$. Normalized dispersion, filtering, and dispersion slope coefficients are $D(z)=-\beta^{\prime \prime}(z) / \beta_{0}^{\prime \prime}, b(z)=B(z) / \beta_{0}^{\prime \prime}$, and $d=-\beta^{\prime \prime \prime} / \beta_{0}^{\prime \prime} T_{0}$, respectively, where $\beta^{\prime \prime \prime}$ is the third-order dispersion and $B(z)$ is the filter curvature. The gain $g(z)$ may be written as

$$
g(z)= \begin{cases}g_{n}, & z_{n}<z<z_{n}+L_{\mathrm{amp}} \\ -\Gamma, & \text { elsewhere }\end{cases}
$$

where $g_{n}$ and $z_{n}$ are the gain coefficient and the position of the $n$th amplifier seen by the pulse, $\Gamma$ is the loss coefficient in the 
fiber, $L_{\text {amp }}$ is the amplifier length. The noise contribution $\hat{F}$ from the amplifiers has the autocorrelation function

$$
\left\langle\hat{F}(z, t) \hat{F}^{*}\left(z^{\prime}, t^{\prime}\right)\right\rangle=2 g_{n} \theta(z) \frac{n_{2} \hbar \omega_{0}^{2} L_{D}}{A_{\mathrm{eff}} T_{0} c} \delta\left(z-z^{\prime}\right) \delta\left(t-t^{\prime}\right)
$$

where $\theta(z)$ is the spontaneous emission factor $n_{\mathrm{sp}}=1.3$ when $z_{n}<z<z_{n}+L_{\mathrm{amp}}$, and we set $\theta(z)=0$ elsewhere. To accurately calculate the interpulse interaction in the transient regime we model the saturated gain dynamics using the rate equation

$$
\frac{\partial g_{n}}{\partial t}+\frac{g_{n}-g_{0}}{\tau_{a}}=-\frac{g_{n}|q|^{2}}{\tau_{a} P_{\text {sat }}}
$$

where $\tau_{a}$ is the relaxation time of the amplifier set to $1 \mathrm{~ms}$ and $P_{\text {sat }}$ is the saturation power of the amplifier set to $10 \mathrm{~mW}$. While these values do not precisely equal the experimental values, and, consequently, the details of the transient evolution are somewhat different in the experiment and the theory [9], the extinction ratios that we calculate are not sensitive to these choices.

To study the interpulse interactions in our simulations, we propagate two pulses that are separated by $100 \mathrm{ps}$ in the case of our studies at $10 \mathrm{Gbit} / \mathrm{s}$ and by $50 \mathrm{ps}$ in the case of our studies at $20 \mathrm{Gbit} / \mathrm{s}$. Our propagation distance is $24500 \mathrm{~km}$, corresponding to the maximum experimental value [7], [10]. We choose Gaussian initial pulses having long trailing tails at large positive times such that

$$
q(0, t)=A \exp \left[-4(t / \tau)^{2}\right]+B \operatorname{sech}(t / 4 \tau)
$$

at $t>0$ and

$$
q(0, t)=(A+B) \exp \left[-4(t / \tau)^{2}\right]
$$

at $t \leq 0$. We vary the extinction ratio of the pulse by changing $B$, and we vary the pulse duration by changing $\tau$. For a 20-ps pulse, $\tau$ equals 11.34 ps. We note that our initial condition is asymmetric, which is consistent with our experiments as we describe later. However, we have found that replacing this asymmetric initial condition with a symmetric initial condition in which

$$
q(0, t)=A \exp \left[-4(t / \tau)^{2}\right]+B \operatorname{sech}(t / 4 \tau)
$$

for all $t$ has little effect on the results. We choose a shift of 5.6 ps as the criterion for safe transmission at 10-Gbit/s data rate as the central time standard deviation of $5.6 \mathrm{ps}$ corresponds to a bit error rate of $10^{-9}$ with a 100-ps time slot, assuming an $80 \%$ safety window and a Gaussian distribution of the time shifts. We set the transmission distance to $24500 \mathrm{~km}$ when the shift due to the interpulse interaction remains smaller than $5.6 \mathrm{ps}$. Although for 20-Gbit/s transmission the safe standard deviation of the central time is smaller than $5.6 \mathrm{ps}$, we still use the same criterion in order to compare the strength of the interpulse interaction at 10- and 20-Gbit/s transmission.

\section{Simulation Results}

Fig. 2 shows the dependence of the transmission distance on the extinction ratio at $50 \mathrm{ps}$ from the pulse peak in a loop with a path average dispersion of $0.02 \mathrm{ps} / \mathrm{nm} \cdot \mathrm{km}$. In Fig. 2(a), we see that there is a remarkable tolerance of the transmission distance to the pulse tails up to $-13 \mathrm{~dB}$ at $50 \mathrm{ps}$ when the interpulse separation is $100 \mathrm{ps}$, corresponding to a data rate of 10

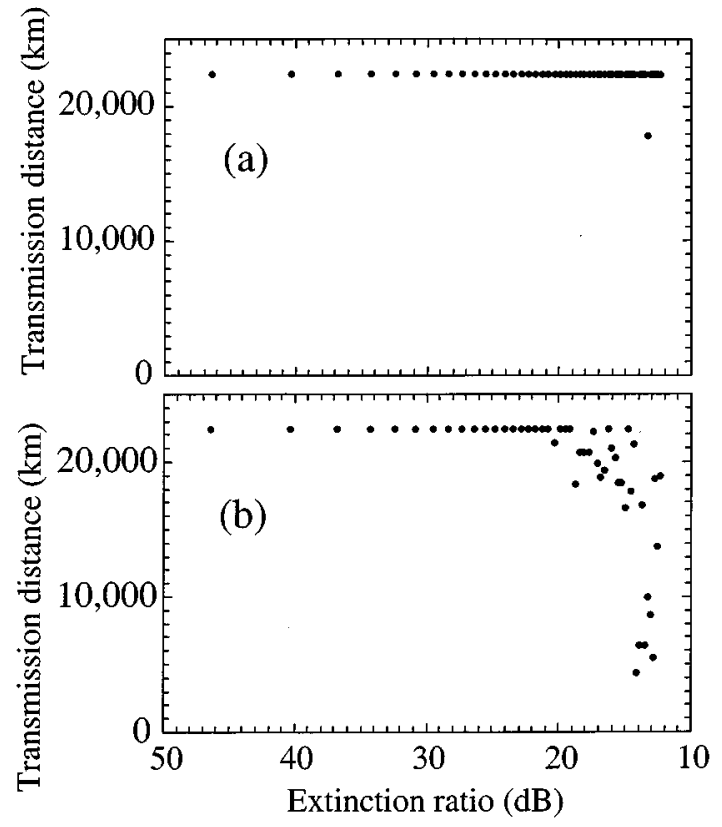

Fig. 2. Dependence of the transmission distance on the extinction ratio $50 \mathrm{ps}$ from the peak at (a) 100-ps pulse separation corresponding to $10-\mathrm{Gbit} / \mathrm{s}$ data transmission and (b) 50-ps pulse separation corresponding to 20-Gbit/s data transmission, with an anomalous path average dispersion of $0.02 \mathrm{ps} / \mathrm{nm} \cdot \mathrm{km}$. The pulse durations in this case are $20 \mathrm{ps}$.

Gbits/s. We varied the pulse durations in a range of $15-25 \mathrm{ps,}$ obtaining little change in the results. Hence, an extinction ratio of $15 \mathrm{~dB}$ is certainly sufficient. However, when the interpulse separation is $50 \mathrm{ps}$, corresponding to a data rate of $20 \mathrm{Gbits} / \mathrm{s}$, as shown in Fig. 2(b), the transmission distance drops significantly when the tails at $50 \mathrm{ps}$ from the peak becomes larger than $-19 \mathrm{~dB}$ due to stronger overlap of the pulses. Thus the extinction ratio should be larger than about $20 \mathrm{~dB}$. Again, varying the pulse durations over a range of 15-25 ps made little difference in the results. The spread of the data in Fig. 2 occurs because the power level of the spontaneous emission noise nearly equals that of the tail at the point where it overlaps the neighboring pulse. Thus the strength of the interaction varies by almost $100 \%$ from case to case. When the path average dispersion becomes slightly normal, the stretching factor of the DMS significantly increases [11], thus increasing the overlap of the adjacent pulses. For this reason, the transmission distance degrades far more rapidly than when the dispersion is in the anomalous dispersion regime, as shown in Fig. 3. With a 100-ps pulse separation and a normal path average dispersion of $-0.01 \mathrm{ps} / \mathrm{nm} \cdot \mathrm{km}$, the transmission distance does not exceed $7000 \mathrm{~km}$ when the extinction ratio is $15 \mathrm{~dB}$. In order to achieve propagation over the full distance of $24500 \mathrm{~km}$, the extinction ratio must be at least $25 \mathrm{~dB}$. In the normal dispersion regime, the pulse durations must be shorter than in the anomalous dispersion regime, and the powers must be substantially higher. We varied the initial pulse durations in the range of $6-15 \mathrm{ps}$ with little change in the results. We note that there is much less case-to-case variation in Fig. 3 than in Fig. 2(b), indicating that the spontaneous-signal beat noise does not play an important role. Instead, the increased nonlinear interaction due to the large stretching factor dominates the observed behavior. 


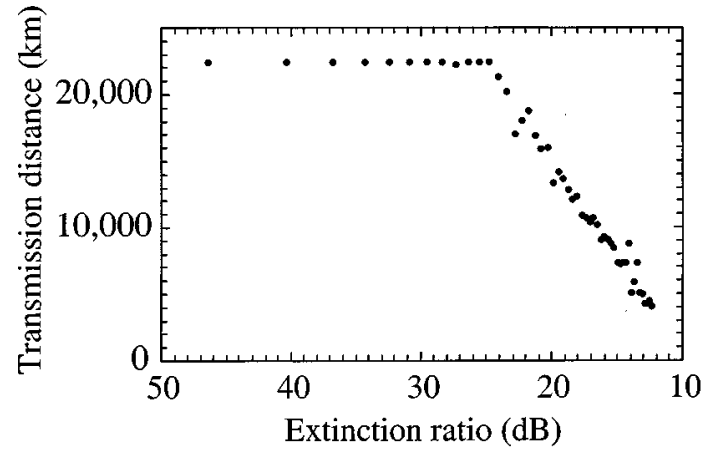

Fig. 3. Dependence of the transmission distance on the extinction ratio at 50 ps from the peak at 100-ps pulse separation corresponding to $10-\mathrm{Gbit} / \mathrm{s}$ data transmission, with a normal path average dispersion of $-0.01 \mathrm{ps} / \mathrm{nm} \cdot \mathrm{km}$. The pulse durations in this case are 6 ps.

\section{EXPERIMENTAL COMPARISON}

We carried out our recirculating loop experiments with two different sources. Our earlier experiments at $10 \mathrm{Gbits} / \mathrm{s}$ were done using a source that consists of a $\mathrm{LiNbO}_{3}$ amplitude modulator followed by a pulse compressor. However, the extinction ratio in this case is only $15 \mathrm{~dB}$, and it was not possible for us to use it to successfully propagate signals at $20 \mathrm{Gbits} / \mathrm{s}$. Moreover, these pulses fail to propagate at $10 \mathrm{Gbits} / \mathrm{s}$ in a loop with zero or slightly normal path average dispersion [11]. Fig. 4(a) shows the pulse shape in this case. In addition to a poor extinction ratio, the pulse is noticeably asymmetric.

We carried out our later experiments using a Pritel fiber laser that generated high-quality pulses with nearly Gaussian pulse shapes. We show the autocorrelation trace in Fig. 4(b). In this case, the extinction ratio is better than $50 \mathrm{~dB}$, allowing us to successfully transmit data at $20-\mathrm{Gbit} / \mathrm{s}$ rate for more than 20 $000 \mathrm{~km}$ in our recirculating loop [10]. In addition to this, using this source, we were able to demonstrate for the first time the long-distance transmission of DMS pulses at zero and slightly normal path average dispersion over $28000 \mathrm{~km}$ [11].

\section{CONCLUSION}

We have shown that long-distance propagation of DMS data can be severely limited by the tails of the source pulses because the interpulse interaction in the transient regime when the input pulses have a poor extinction ratio is significantly stronger than the interpulse interaction in the steady-state DMS regime. We have found that an extinction ratio of $15 \mathrm{~dB}$ at 50 ps from the peak, as obtained from a phase-modulated and filtered source, is sufficient to successfully propagate DMS pulses at $10 \mathrm{Gbits} / \mathrm{s}$ in the anomalous dispersion regime. However, it is not sufficient to propagate signals at $20 \mathrm{Gbits} / \mathrm{s}$ in the anomalous dispersion regime, which requires an extinction ratio of $20 \mathrm{~dB}$ at $50 \mathrm{ps}$ from the peak, and it is not sufficient to propagate signals at $10 \mathrm{Gbits} / \mathrm{s}$ in the normal dispersion regime, which requires an extinction ratio of $25 \mathrm{~dB}$ at $50 \mathrm{ps}$ from the peak. By contrast, a Pritel fiber laser source with an extinction ratio of $50 \mathrm{~dB}$ allows
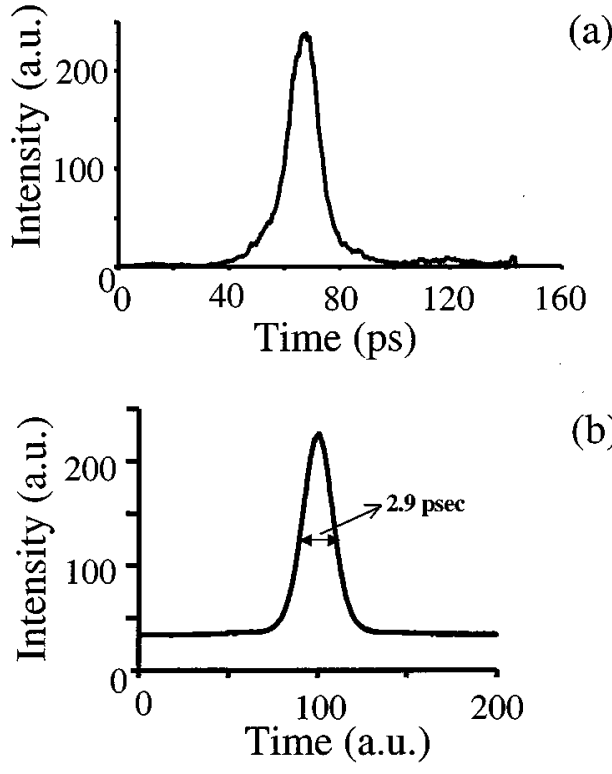

(b)

Fig. 4. (a) Initial pulse shape with which we achieved $28000 \mathrm{~km}$ of error-free propagation at $10 \mathrm{Gbits} / \mathrm{s}$ [7] but with which it was not possible to achieve long-distance propagation at $20 \mathrm{Gbits} / \mathrm{s}$. (b) Autoccrrelation trace of the initial pulse shape with which we achieved $20000 \mathrm{~km}$ of error-free transmission at $20-\mathrm{Gbit} / \mathrm{s}$ data rate [10]. Note the nonzero dc level corresponding to zero power in the tails as expected for an autocorrelation trace that is not background-free.

us to successfully propagate pulses long distances in these two latter cases. We have verified these predictions experimentally.

\section{REFERENCES}

[1] T. Yu, E. A. Golovchenko, A. N. Pilipetski, and C. R. Menyuk, "Dispersion-managed soliton interactions in optical fibers," Opt. Lett., vol. 22, pp. 793-795, 1997.

[2] M. Matsumoto, "Analysis of interaction between stretched pulses propagating in dispersion-managed fibers," IEEE Photon. Technol. Lett., vol. 10, pp. 373-375, 1998.

[3] S. Kumar, M. Wald, F. Lederer, and A. Hasegawa, "Soliton interaction in strongly dispersion-managed optical fibers," Opt. Lett., vol. 23, pp. 1019-1021, 1998.

[4] T. George, "Extended path-averaged soliton regime in highly dispersive fibers," Opt. Lett., vol. 22, pp. 679-681, 1997.

[5] G. Bento, F. Neddam, and S. Wabnitz, "Role of adjacent-pulse overlap in the interaction between dispersion-managed solitons," Opt. Lett., vol. 25, pp. 144-146, 2000.

[6] P. V. Mamyshev and N. A. Mamysheva, "Pulse-overlapped dispersionmanaged data transmission and intrachannel four-wave mixing," Opt. Lett., vol. 24, pp. 1454-1456, 1999.

[7] J. M. Jacob, E. A. Golovchenko, A. N. Pilipetskii, G. M. Carter, and C. R. Menyuk, "Experimental demonstration of soliton transmission over $28 \mathrm{Mm}$ using mostly normal dispersion fiber," IEEE Photon. Technol. Lett., vol. 9, pp. 130-132, 1997.

[8] H. Toda, M. Mino, Y. Kodama, A. Hasegawa, and P. A. Andrekson, "Influence of noise in optical pulse source on soliton transmission," in Proc. Eur. Conf. Optical Communication 1998, Madrid, Spain, pp. 91-92.

[9] R.-M. Mu, V. S. Grigoryan, C. R. Menyuk, G. M. Carter, and J. M. Jacob, "Comparison of theory and experiment for dispersion-managed solitons in a recirculating fiber loop," IEEE J. Select. Topics Quantum Electron., vol. 6, pp. 248-257, Mar./Apr. 2000.

[10] G. M. Carter, R. M. Mu, V. S. Grigoryan, C. R. Menyuk, P. Sinha, T. F. Carruthers, M. L. Dennis, and I. N. Duling III, "Transmission of dispersion-managed solitons at $20 \mathrm{Gbit} / \mathrm{s}$ over $20000 \mathrm{~km}$," Electron. Lett., vol. 35, pp. 133-134, 1999.

[11] V. S. Grigoryan, R.-M. Mu, G. M. Carter, and C. R. Menyuk, "Experimental demonstration of long-distance dispersion-managed soliton propagation at zero average dispersion," IEEE Photon. Technol. Lett., vol. 12 , pp. $45-46$, Jan. 2000. 